\title{
BMJ Open Use of Patient-Reported Outcomes Measurement Information System (PROMIS) measures to characterise health status for patients seeking care from an orthopaedic provider: a retrospective cohort study
}

\author{
Maggie E Horn (DD , ,2 Emily K Reinke, ${ }^{1}$ Xiaofang Yan, ${ }^{3}$ Sheng Luo, ${ }^{4}$ \\ Michael Bolognesi, ${ }^{1}$ Bryce B Reeve, ${ }^{2}$ Steven Z George, ${ }^{1,3}$ Comprehensive \\ Outcomes in Orthopaedics and Rehabilitation Data System (COORDS) group
}

To cite: Horn ME, Reinke EK, Yan $\mathrm{X}$, et al. Use of PatientReported Outcomes Measurement Information System (PROMIS) measures to characterise health status for patients seeking care from an orthopaedic provider: a retrospective cohort study. BMJ Open 2021;11:e047156. doi:10.1136/ bmjopen-2020-047156

- Prepublication history for this paper is available online. To view these files, please visit the journal online (http://dx.doi. org/10.1136/bmjopen-2020047156).

Received 19 November 2020 Accepted 03 August 2021

Check for updates

(c) Author(s) (or their employer(s)) 2021. Re-use permitted under CC BY-NC. No commercial re-use. See rights and permissions. Published by BMJ.

For numbered affiliations see end of article.

Correspondence to

Dr Maggie E Horn;

maggie.horn@duke.edu

\section{ABSTRACT}

Objectives Characterise the health status of patients newly consulting an orthopaedic specialist across eight clinical subspecialties.

Design Retrospective cohort.

Setting 18 orthopaedic clinics, including 8 subspecialties (14 ambulatory and 4 hospital based) within an academic health system.

Participants 14910 patients consulting an orthopaedic specialist for a new patient consultation who completed baseline Patient-Reported Outcomes Measurement Information System (PROMIS) measures associated with their appointment from 17 November 2017 to 13 May 2019. Patients were aged $55.72 \pm 5.8$ years old, and $61.3 \%$ were female and $79.3 \%$ were Caucasian and $13.4 \%$ were black or African American. Patients who did not complete PROMIS measures or cancelled their appointment were excluded from the study.

Primary outcome PROMIS domains of physical function, pain interference, pain intensity, depression, anxiety, fatigue, sleep disturbance and the ability to participate in social roles.

Results Mean PROMIS scores for physical function were (38.1 \pm 9.2$)$, pain interference (58.9 \pm 8.1$)$, pain intensity

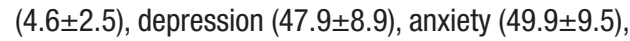
fatigue (50.5 \pm 10.3$)$, sleep disturbance (51.1 \pm 9.8$)$ and ability to participate in social roles $(49.1 \pm 10.3)$ for the entire cohort. Across the clinical subspecialties, neurosurgery, spine and trauma patients were most profoundly affected across almost all domains and patients consulting with a hand specialist reported the least limitations or symptoms across domains. There was a moderate, negative correlation between pain interference and physical functioning $(r=-0.59)$ and low correlations between pain interference with anxiety $(r=0.36)$, depression ( $r=0.39$ ) as well as physical function and anxiety $(r=-0.32)$ and depression $(r=-0.30)$ and sleep $(r=-0.31)$.

Conclusions We directly compared clinically meaningful PROMIS domains across eight orthopaedic subspecialties,
Strengths and limitations of this study

- This study demonstrated the direct comparison of health status using Patient-Reported Outcomes Measurement Information System (PROMIS) measures across eight orthopaedic clinical subspecialties, which was previously a challenge using legacy outcome measures.

- This study reported the association of eight clinically relevant PROMIS domains (six physical health and two mental health domains) within an orthopaedic cohort.

- We evaluated PROMIS measures at baseline only as part of routine clinical assessment associated with a new patient consultation with an orthopaedic specialist; no follow-up data were analysed in the context of downstream healthcare utilisation.

- The findings' generalisability is limited by data collected within a private health system setting that may not reflect other health systems' characteristics.

which would not have been possible with legacy measures alone. These results support PROMIS's utility as a common metric to assess and compare patient health status across multiple orthopaedic subspecialties.

\section{INTRODUCTION}

To determine if a patient has achieved treatment success, it is insufficient to evaluate treatment results solely on medical history, physical findings, laboratory tests or imaging findings alone. ${ }^{1}$ While these are essential clinical indicators, they may not reflect what is most important to a patient. Patient-reported outcome measures (PROMs) are additional indicators that come directly from the patient. PROMs may address more important 
patient-centred outcomes about a patient's health status's physical, mental and social aspects. Change in health status can be one of the measures of 'success' from a patient's perspective after an orthopaedic procedure. ${ }^{2}$ PROMs are increasingly being used as part of the clinical encounter to guide treatment decisions and determine intervention effectiveness. ${ }^{3}$

'Legacy' patient-reported outcome measures (PROMs) have been used for decades; however, they have many limitations. ${ }^{4}$ To overcome the limitations of legacy measures, the NIH developed a universally accepted set of PROMs. The NIH's Patient-Reported Outcomes Measurement Information System (PROMIS) covers a broad range of relevant domains and has strong evidence for its validity and reliability in a broad range of populations. ${ }^{5-8}$ In orthopaedics, the use of PROMIS measures has distinct advantages because it can be used across many clinical subspecialties as a common outcome metric. ${ }^{8-10}$ This has the opportunity to allow for the evaluation of the efficacy of different interventions and inform quality improvement initiatives. ${ }^{11}$

Recently, there has been an increase in the adoption of PROMIS measures as the standard outcome measurement system in orthopaedics to assess health status in orthopaedic patients. ${ }^{8}$ However, what is unknown about the use of PROMIS measures in orthopaedics is how these measures differ across patients seeking care from different orthopaedic clinical subspecialties. Moreover, there are limited data regarding the burden of disease in patients presenting to orthopaedic clinics for initial care. Therefore, there are two goals of this study. First, we will characterise the health status of a cohort of patients completing PROMIS measures as part of the clinical encounter by comparing the physical health (six domains) and mental health (two domains) across eight different clinical subspecialty areas in a large academic medical centre. Second, we will examine the correlation between the PROMIS domains in this cohort.

\section{METHODS}

\section{Study setting and participants}

Patients consulting an orthopaedic specialist (surgeon or advanced practice provider-nurse practitioner or physician assistant) for a new patient consultation from 17 November 2017 to 13 May 2019 were considered. In this study, patients sought care within the department of orthopaedic surgery at a large academic, private medical centre in Durham, North Carolina. Inclusion criterion for the study was patients who completed assigned PROMIS measures associated with the visit type of new patient appointment. We excluded patients from the study who (1) were under 18 years of age at time of appointment, (2) completed assigned PROMIS measures but cancelled or did not attend their scheduled appointments and (3) who attended a visit with a provider that was not classified as an orthopaedic specialist or a provider that did not have a provider specialty designation within the electronic

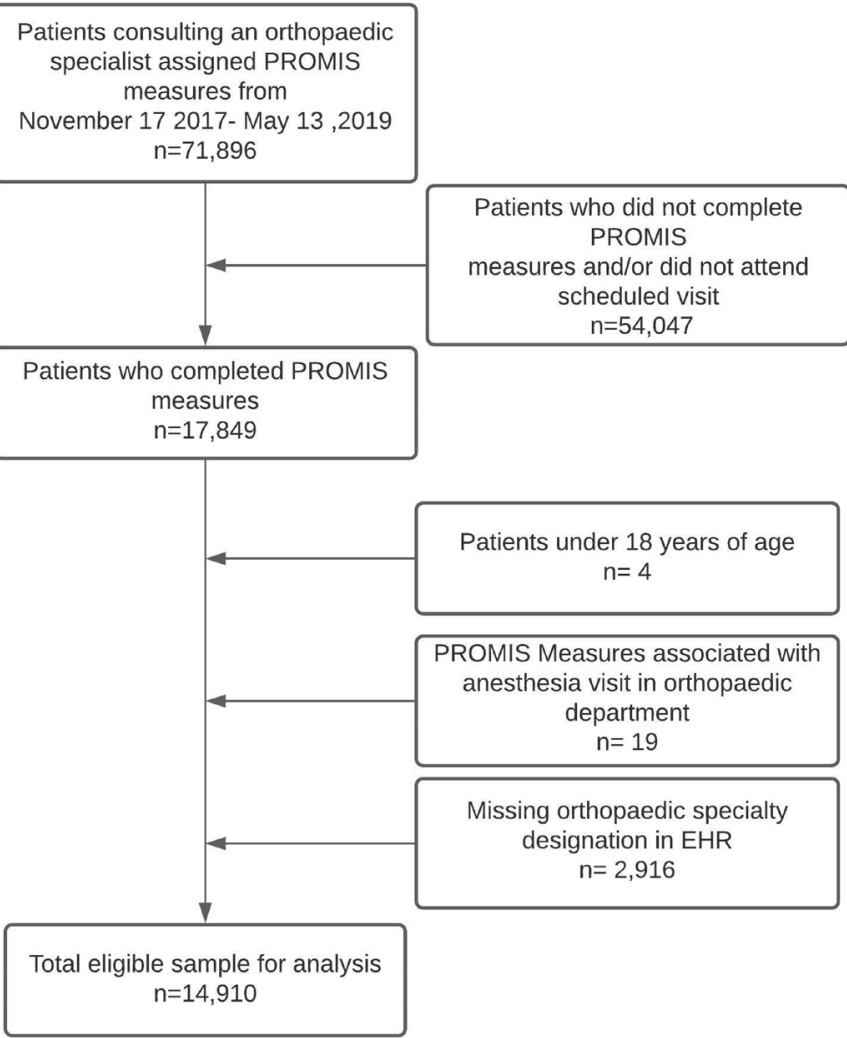

Figure 1 Flow diagram for cohort selection. EHR, electronic health record; PROMIS, Patient-Reported Outcomes Measurement Information System.

health record (EHR). The department includes 18 adult clinics (14 ambulatory and 4 hospital-based clinics). See figure 1 for study eligibility. The department consists of eight subspecialties (joint reconstruction, spine, neurosurgery, sports medicine, trauma, orthopaedic oncology, foot and ankle and hand) with over 100 orthopaedic specialists. We extracted all data for this study directly from the EHR.

\section{Standardised collection of PROMIS measures}

In December 2017, the orthopaedics department implemented a standardised collection of PROMIS measures across 18 clinics and 8 clinical subspecialties. The administration of PROMIS measures was linked to new patient appointments and collected and scored passively within the EHR (Epic Systems) as part of the standard of care. Therefore, informed consent was not required for the completion of the PROMIS measures.

From November 2017 to May 2019, we collected the short-form version of the following eight PROMIS domains: physical function (seven Items), pain interference (eight items), pain intensity (one item), depression (eight items), anxiety (eight items), fatigue (eight items), sleep disturbance (eight items) and ability to participate in social roles (eight items). On 20 December 2018, our health system transitioned to administering the computer adaptive testing (CAT) version of PROMIS domains instead of short form as the CAT instruments were then available within our EHR. As part of the transition 
from short form to CAT, we reduced the set of PROMIS domains collected to physical function, pain interference, depression, and sleep disturbance. This change was done in response to feedback from clinicians regarding respondent burden with eight domains and perceived clinical utility of information gained. Studies to date suggest that CATs andshort forms will produce very similar mean scores for a given sample and demonstrate that similar accuracy range withshort forms is greater than four items such as in this study. ${ }^{12-14}$ Therefore, we combined these scores with the respective PROMIS short form scores for the analysis.

The PROMIS physical function domain is a patient's self-reported capability (rather than actual performance) of physical activities. The physical function domain includes the functioning of one's upper extremities (dexterity), lower extremities (walking or mobility) and central regions (neck, back), as well as instrumental activities of daily living, such as running errands. ${ }^{15}$ PROMIS pain interference measures the consequences of pain on relevant aspects of one's life. The pain interference domain includes the extent to which pain hinders engagement with social, cognitive, emotional, physical and recreational activities. ${ }^{16}$ PROMIS pain intensity consists of one question, 'How would you rate your pain on average? $\left(0-10,0=\right.$ no pain $10=$ worst imaginable) ${ }^{1}{ }^{17}$ PROMIS emotional distress domains included depression and anxiety. Depression measures negative mood (sadness, guilt), views of self (self-criticism, worthlessness) and social cognition (loneliness, interpersonal alienation), as well as decreased positive affect and engagement (loss of interest, meaning and purpose).$^{18}$ Anxiety domain measures fear (fearfulness, panic), anxious misery (worry, dread), hyperarousal (tension, nervousness, restlessness) and somatic symptoms related to arousal (racing heart, dizziness) ${ }^{19}$ The PROMIS sleep disturbance perceptions measure sleep quality, sleep depth and restoration associated with sleep. ${ }^{20}$ The PROMIS fatigue domain measures a range of symptoms, from mild subjective feelings of tiredness to an overwhelming, debilitating and sustained sense of exhaustion that likely decreases one's ability to execute daily activities and function normally in a family or social roles. ${ }^{21}$ The PROMIS ability to participate in social roles and activities measures one's perceived ability to perform one's usual social roles and activities. ${ }^{22} 23$

Each PROMIS domain is scored separately on a T-score metric, where 50 is the mean and 10 is the SD of the calibration population. For all PROMIS domains (except sleep disturbance) included in this study, the calibration population is the US general population. A higher score on a domain reflects more of the measured concept (eg, more fatigue, more physical function). For example, a physical function score of 60 indicates that the sample's functioning is $1 \mathrm{SD}$ better than the average US general population and lower score is less of the measured concept. ${ }^{5}$ To increase the interpretability of findings, PROMIS scores can then be categorised into the categories of 'within normal limits' indicating less than a
$0.5 \mathrm{SD}$ from the mean; 'mild' indicating a score $0.5 \mathrm{SD}$ from mean; 'moderate' indicating 1.0 SD from the mean and 'severe' indicating 2.0 SD from the mean. These categories were developed by evaluating the percentage of participants from large-scale calibration testing that would then fit into each category. ${ }^{10} 24$

\section{Patient demographics}

Patient demographics recorded included patient age at the appointment, sex (male or female), race (American Indian or Alaskan Native, Asian, black or African American, Caucasian/white, Native Hawaiian or other Pacific Islander, not reported/declined, other and two or more races), ethnicity (Hispanic, non-Hispanic/nonLatino, not reported/declined), marital status (divorced, legally separated, life partner, married, single, unknown, widowed), geographical delineation (urban or rural) and primary and secondary insurance type (Medicare, Medicaid, workers compensation, private).

\section{Healthcare process variables}

In this sample, we collected information related to the new patient consultation, including visit date, clinic location and type (ambulatory vs hospital based), provider type (orthopaedic physician or advanced practice provider-nurse practitioner or physician assistant) and provider specialty (joint reconstruction, spine, neurosurgery, sports medicine, trauma, orthopaedic oncology, foot and ankle and hand).

\section{Data analysis}

We performed data analysis using $\mathrm{R}$ statistical software V.R $4.0 .2^{25}$

This study's primary purpose was to characterise the health status of patients seeking care from eight orthopaedic subspecialties in the department of orthopaedic surgery. We calculated descriptive statistics to characterise the cohort. Means and SD were reported for continuous variables and percentages were reported for categorical variables. Cohort characteristics were compared across clinical subspecialties using $\chi^{2}$ analysis for categorical variables and one-way analyses of variance for continuous variables. We conducted ordinary least squares linear regression and reported the mean and 95\% CI SD for PROMIS domain scores across each specialty and for the entire cohort. We included the variables of age, sex (male, female), race (collapsed into Caucasian/white, black/ African American and other), ethnicity (collapsed into non-Hispanic, Hispanic and not reported) and instrument type (short form or CAT). These variables were included to control for the effects of differences in demographic factors and questionnaire type across specialities when comparing mean PROMIS scores. We then calculated the percentage of patients in the total cohort and each clinical subspecialty by severity categories for each PROMIS domain: within normal limits, mild, moderate and severe. ${ }^{102426}$ Lastly, we performed Pearson correlation analyses to determine the association of the eight 
PROMIS domains in the cohort. We defined the magnitude of correlation as follows: low correlation-0.10-0.39, moderate correlation- $0.40-0.69$, high correlation-0.70-0.89 and very high correlation-0.90-1.00. ${ }^{27}$

\section{Patient and public involvement}

There was no involvement from patients or members of the public in the design, or conduct, or reporting, or dissemination plans of this study.

\section{RESULTS}

\section{Cohort demographics}

Our study included 14910 patients who consulted an orthopaedic specialist for a new patient consultation and completed baseline PROMIS measures. Of the entire sample, $61.3 \% \quad(\mathrm{n}=9137)$ were female with a mean age of 55.72 (15.8) years. Most of the samples reported being Caucasian $(79.3 \% \quad(\mathrm{n}=11 \quad 831))$ and $13.4 \% \quad(\mathrm{n}=2001)$ were black or African American. Our sample's selfreported ethnicity was $93.7 \%$ ( $\mathrm{n}=13$ 976) non-Hispanic/ non-Latino and $1.9 \%(\mathrm{n}=285)$ Hispanic. The majority of patients in the sample were married $(64.1 \%, \mathrm{n}=9553)$. The geographic dispersion of the sample included $83.8 \%$ $(n=12488)$ residing in urban areas and $8.6 \%(n=1276)$ residing in rural areas in North Carolina. The primary insurance of the sample was predominantly private $(90.3 \%, \mathrm{n}=13 \mathrm{465})$ followed by Medicare $(7.3 \%, \mathrm{n}=1088)$ and Medicaid (2.1\%, $\mathrm{n}=311)$.

The highest volume of patients in the sample sought care from a sports medicine provider $(28.1 \%, \mathrm{n}=4197)$ or a spine provider $(20.3 \%, \mathrm{n}=3028)$, followed by total joint $(15.8 \%, \mathrm{n}=2353)$, foot and ankle $(14.8 \%, \mathrm{n}=2208)$ and hand $(12.5 \%, \mathrm{n}=1858)$. Orthopaedic oncology, neurosurgery and trauma had fewer than $10 \%$ of the total volume of patients in the sample, see table 1.

\section{PROMIS scores}

As presented in table 2, the adjusted mean scores for the PROMIS domains for the entire cohort were 38.14 $(38.00,38.28)$ for physical function, $58.84(58.71,58.98)$ for pain interference, $4.57(4.53,4.62)$ for pain intensity (on a $0-10$ scale), $47.87(47.73,48.01)$ for depression, $49.85(49.67,50.03)$ for anxiety, $50.49(50.29,50.68)$ for fatigue, $51.08(50.92,51.24)$ for sleep disturbance and 49.06 (48.86, 49.25) for ability to participate in social roles. Higher pain interference and lower physical function were at least 0.5 and $-1.0 \mathrm{SD}$ away, respectively, from the average US general population.

Table 3 provides more context to the range of observed health status scores by categorising scores into degrees of severity: within normal limits, mild, moderate and severe. ${ }^{102426}$ In this cohort, $24.9 \%$ of all patients reported their physical functioning within normal limits, the majority of patients $(75 \%)$ reported mild, moderate or severe limitations in physical functioning. There is a similar trend for pain interference, where $73 \%$ of patients reported mild, moderate or severe limitations with pain interference. The majority of the cohort reported within normal limits for the ability to participate in social roles $(63.5 \%)$, fatigue $(68.0 \%)$ and sleep disturbance $(66.0 \%)$. For the mental health domains (anxiety and depression), across the samples, most patients reported normal limits for anxiety $(69.6 \%)$ and depression $(76.8 \%)$. Few patients reported severe symptoms of anxiety $(1.7 \%)$ and depression $(1.1 \%)$.

In table 4, we examine the correlation of unadjusted PROMIS domains in the cohort. As expected, we found high correlations between depression and anxiety $(\mathrm{r}=0.76)$ and pain interference and pain intensity $(\mathrm{r}=0.75)$. Additionally, we found moderate correlations between commonly administered PROMIS domains in orthopaedics-physical function and pain interference $(\mathrm{r}=-0.60)$ and physical function and pain intensity $(-0.52)$. There was a low correlation found between anxiety and pain intensity $(\mathrm{r}=0.30)$, anxiety and pain interference $(\mathrm{r}=0.36)$ and anxiety and physical function $(\mathrm{r}=-0.32)$. Similar trends were found with depression where there were low correlations between depression and pain intensity $(r=0.29)$, depression and pain interference $(r=0.39)$ and depression and physical function $(\mathrm{r}=-0.30)$.

\section{PROMIS scores by major clinical specialties}

Foot and ankle

Patients consulting with a foot and ankle orthopaedic specialist scored less than 0.5 SD from the mean for all domains except for pain interference, where patients reported a mean score of $57.2(8.6)$. When looking at the clinically interpretable categories for physical function, $30.7 \%$ of patients reported moderate limitations and $16.7 \%$ severe limitations. For pain interference, 39.4\% reported moderate limitations and $3.2 \%$ reported severe limitations.

\section{Hand}

Patients who consulted with a hand orthopaedic specialist reported scores less than $0.5 \mathrm{SD}$ from the mean for all domains except for pain interference and physical function. Patients reported a mean score of 55.7 (8.8) and 41.85 (9.5), respectively. For physical function, $26.4 \%$ of patients reported moderate limitations and $12.5 \%$ reported severe limitations.

\section{Neurosurgery and spine}

Patients either consulting with a neurosurgeon or a spine orthopaedic specialist reported between $1 \mathrm{SD}$ and 1.5 SD from the US mean for pain interference and physical functioning. Neurosurgery patients reported higher levels of pain interference (61.53 (8.1)) compared with spine patients (57.23 (9.7)) and also reported more limitations in physical functioning (35.0 (9.0)) as compared with spine patients $(38.35$ (10.5)). For physical function, a majority of neurosurgery patients reported moderate $(38.9 \%)$ and severe $(31.8 \%)$ limitations and this was similar in spine patients (moderate $40.5 \%$, severe $28.8 \%$ ). For pain interference, most neurosurgery $(59.7 \%)$ and 


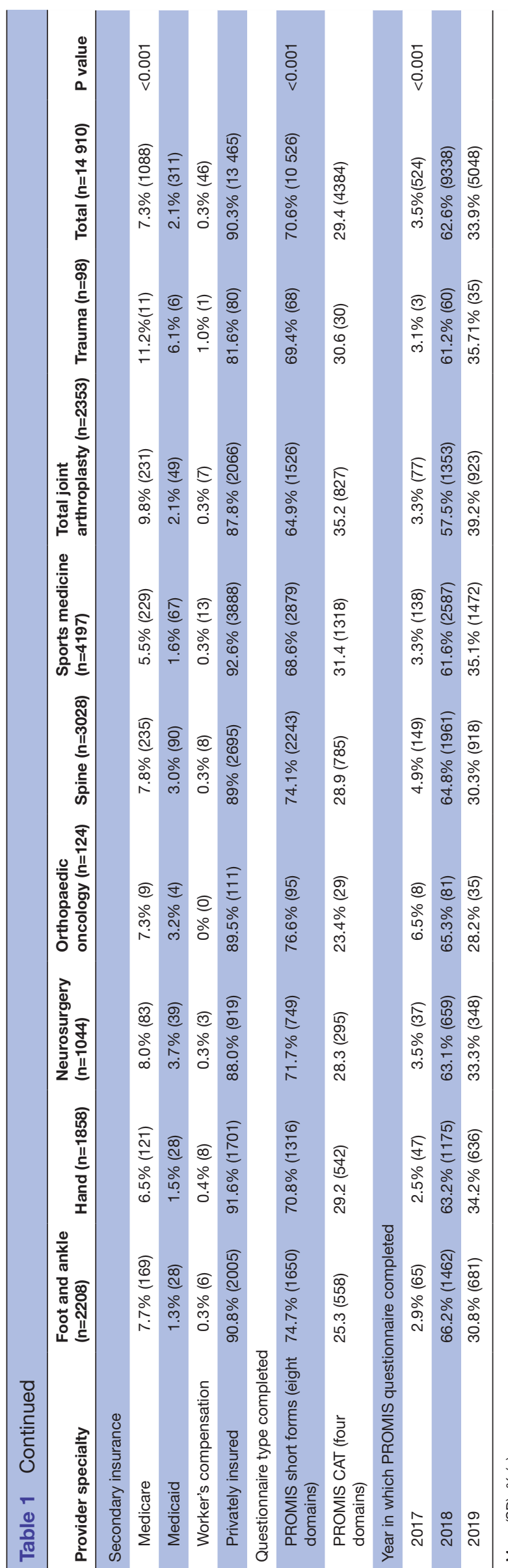

spine patients $(55.8 \%)$ reported moderate limitations and a small percentage of neurosurgery $(7.4 \%)$ and spine patients $(6.2 \%)$ reported severe limitations.

\section{Sports medicine}

Patients seeking care from a sports medicine specialist reported less than $0.5 \mathrm{SD}$ from the US mean in all domains except in physical function (39.51 (8.8)) and pain interference $(58.0(7.6))$ where their scores were between $0.5 \mathrm{SD}$ and 1.0 SD from the US mean. Overall, $33.3 \%$ of patients reported moderate limitations in physical function and $15.4 \%$ reported severe limitations. For pain interference, $38.0 \%$ of patients reported moderate limitations and $3.7 \%$ reported severe limitations.

Total joint arthroplasty

Patients consulting a total joint arthroplasty orthopaedic surgeon reported less than $0.5 \mathrm{SD}$ from the US mean in all domains except in physical functioning (36.58 (8.5)) and pain interference $(60.21$ (8.4)). Most patients reported moderate limitations in physical functioning $(40.2 \%)$ and pain interference $(48.9 \%), 23.2 \%$ reported severe limitations in physical function and only a small percentage reported severe limitations for pain interference $(6.5 \%)$.

We did not report on trauma or orthopaedic oncology due to low sample sizes in each of these subspecialties.

\section{DISCUSSION}

The goal of PROMIS was to create a measurement system that could standardise PROMs across chronic conditions to better enable comparisons across different disease conditions. ${ }^{14}$ To this end, we described approximately 15 000 orthopaedic patients across eight different clinical subspecialties who completed PROMIS measures associated with a new patient consultation with an orthopaedic specialist. We found across an orthopaedic department at an academic medical centre that most patients reported scores within $0.5 \mathrm{SD}$ from the US mean on all domains except on pain interference and physical functioning where they reported approximately 1.0 SD US mean on pain interference and physical functioning. These findings are expected, where the primary drivers of seeking care for orthopaedic issues are decreased physical functioning and increased interference with activities due to pain. ${ }^{28}{ }^{29}$ When further examining the difference of PROMIS scores between the clinical subspecialties for the physical health domains, we found that patients seeking care from hand specialists reported less overall physical health impairments. However, we primarily attribute this finding to administer the generic PROMIS physical function measure rather than the upper extremity physical function PROMIS measure, which is more specific to upper extremity conditions and may better reflect limitations in this group. ${ }^{30}$ Patients seeking care from a neurosurgery, spine or a trauma specialist reported significant physical health impairments. When examining PROMIS mental health domains, most patients across clinical 


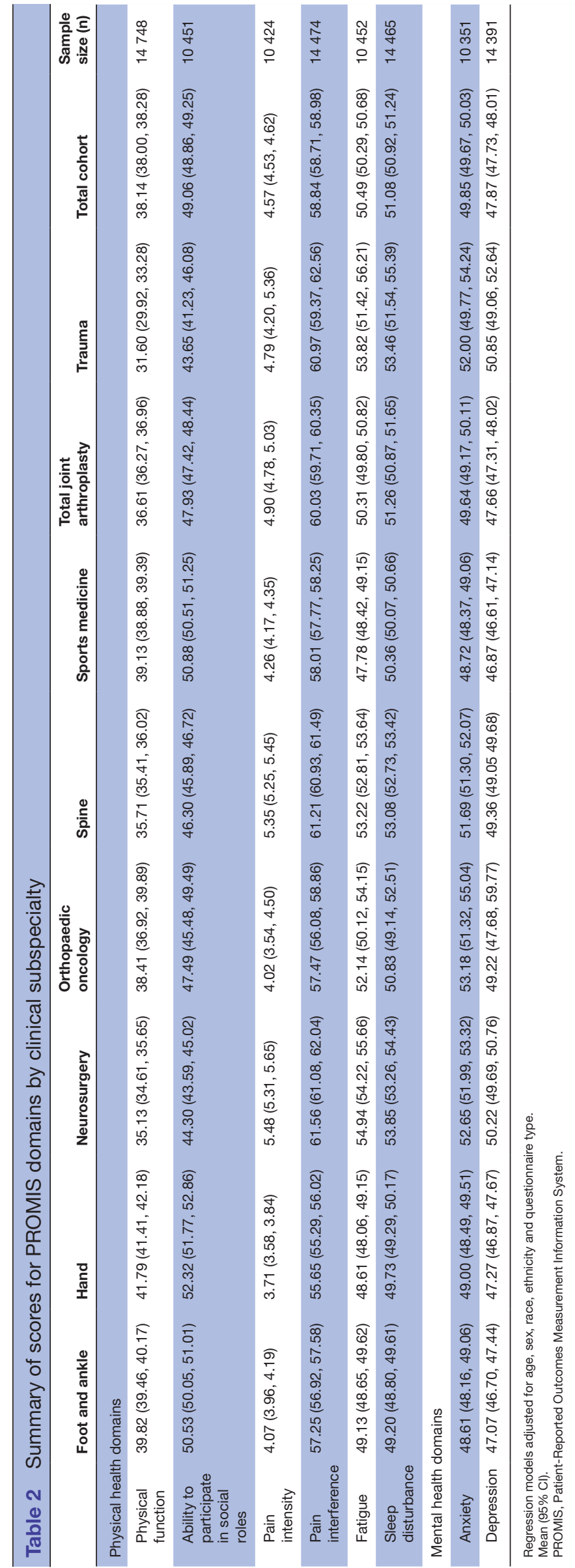


Table 3 PROMIS severity categories by clinical subspecialty

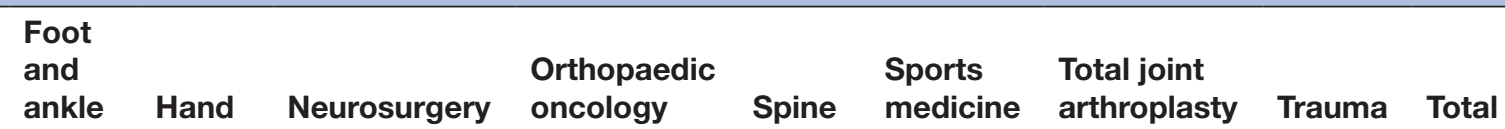

Physical health domains

Physical function

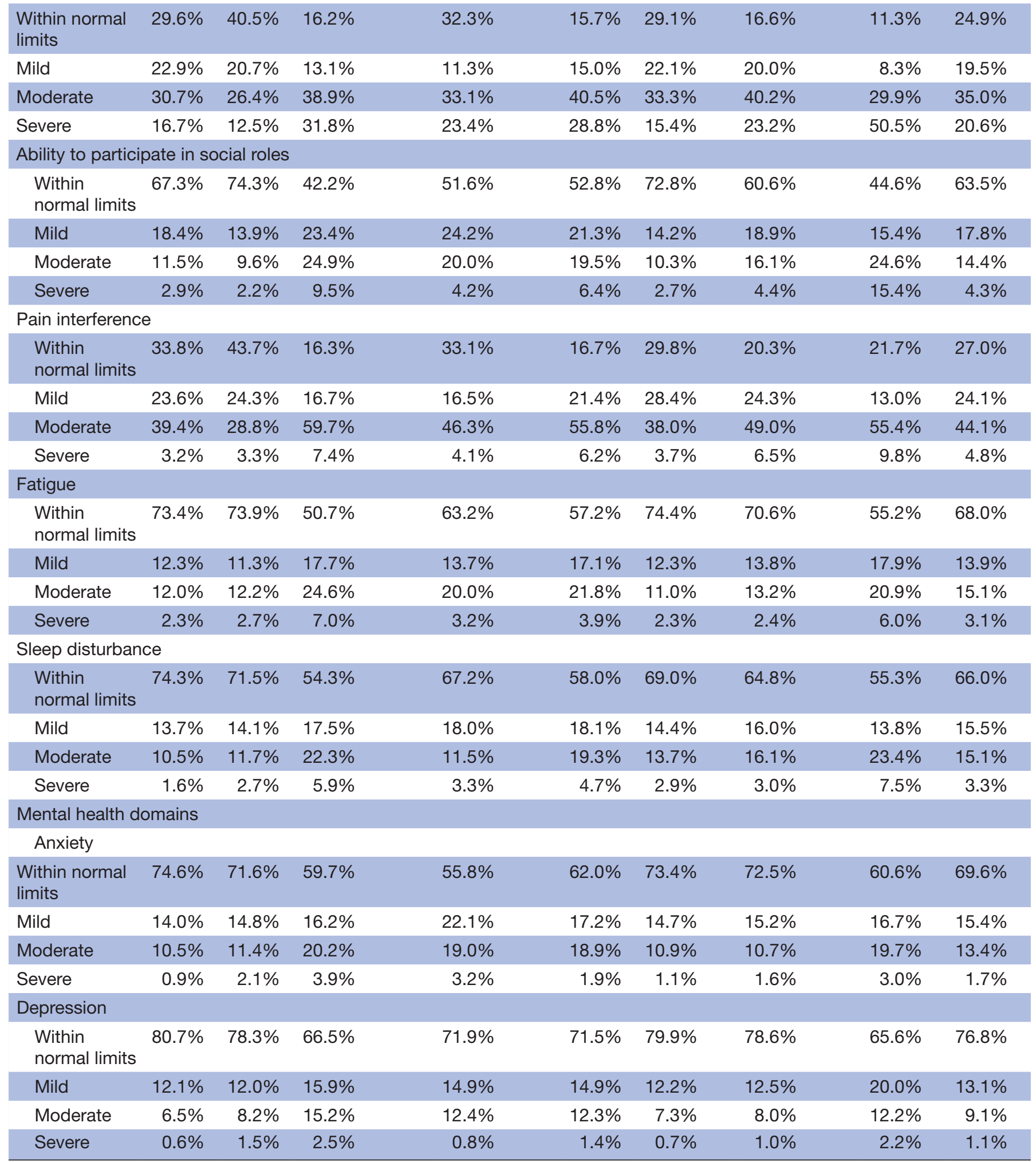

PROMIS, Patient-Reported Outcomes Measurement Information System. 
Table 4 Correlation of PROMIS domains

\begin{tabular}{llccccccc}
\hline & Participation & Anxiety & Depression & Fatigue & $\begin{array}{l}\text { Pain } \\
\text { intensity }\end{array}$ & $\begin{array}{l}\text { Pain } \\
\text { interference }\end{array}$ & $\begin{array}{l}\text { Physical } \\
\text { function }\end{array}$ & $\begin{array}{l}\text { Sleep } \\
\text { disturbance }\end{array}$ \\
\hline Participation & - & -0.48 & -0.51 & -0.66 & -0.52 & -0.69 & 0.67 & -0.45 \\
Anxiety & -0.48 & - & 0.76 & 0.58 & 0.30 & 0.36 & -0.32 & 0.44 \\
\hline Depression & -0.51 & 0.76 & - & 0.58 & 0.29 & 0.39 & -0.30 & 0.43 \\
\hline Fatigue & -0.66 & 0.58 & 0.58 & - & 0.42 & 0.52 & -0.48 & 0.54 \\
Pain intensity & -0.52 & 0.30 & 0.29 & 0.42 & - & 0.75 & -0.52 & 0.39 \\
Pain interference & -0.69 & 0.36 & 0.39 & 0.52 & 0.75 & - & -0.60 & 0.45 \\
Physical function & 0.67 & -0.32 & -0.30 & -0.48 & -0.52 & -0.60 & - & -0.31 \\
\hline Sleep disturbance & -0.45 & 0.44 & 0.43 & 0.54 & 0.39 & 0.45 & -0.31 & - \\
\hline
\end{tabular}

All correlations were statistically significant $p<0.001$.

PROMIS, Patient-Reported Outcomes Measurement Information System.

subspecialties reported that their anxiety and depression symptoms were within normal limits; however, up to $24.1 \%$ reported moderate or severe anxiety and up to $17.6 \%$ reported moderate or severe depressive symptoms. Following a similar trend as the specialties across the PROMIS physical health domains, patients seeking care from a neurosurgery, spine or trauma specialist reported the highest anxiety and depression symptoms. This relationship is consistent with the literature supporting patients with spine conditions and orthopaedic trauma have higher anxiety levels than other orthopaedic conditions. ${ }^{31} 32$

Majority of patients seeking care in orthopaedics across clinical subspecialties do so because of limitations in physical function and pain. ${ }^{33-35}$ Measurement of these constructs can be done using PROMs. Many legacy measures commonly used in orthopaedics measure more than one construct. This makes it difficult to elucidate limitations or symptom contributions from a specific construct on patients perception of their health status. For example, a patient seeking care for knee osteoarthritis may report more limitations in physical functioning rather than pain. Using traditional, concise legacy measures such as Knee Injury and Osteoarthritis Outcome Score Junior (KOOS Jr) ${ }^{36}$ to evaluate stiffness, pain, function and activities of daily living using seven items would be challenging to isolate the relative limitations in physical functioning compared with the other constructs included in the KOOS Jr. Whereas PROMIS physical function can be administered to capture this construct separately and concisely, giving a valid estimate of a patient's perception of their physical function. PROMIS physical function and has been shown to be equal or superior in regard to floor and ceiling effects when compared with previously established legacy PROM in several patient populations including trauma, shoulder, elbow, hand, spine, and knee; making this measure applicable across patient populations and range of severity of symptoms. ${ }^{37}$ However, a noted limitation is, to date, not all PROMIS measures have been evaluated for floor and ceiling effects across multiple populations or found to be as responsive as PROMIS physical function for orthopaedic patients. In particular, PROMIS measures that capture emotional distress and psychosocial illness impact have not been extensively researched for widespread use in orthopaedics. ${ }^{31}$

\section{Strengths and limitations}

Our study has noted strengths. First, our study is novel in that we reported eight PROMIS domains across eight different orthopaedic clinical subspecialties. The use of PROMIS measures in clinical practice and research has been increasing in prevalence in spine, total joint, sports medicine, upper extremity disorders, trauma and lower extremity disorders ${ }^{38}$ In a systematic review on the uptake of PROMIS measures in orthopaedics, Horn et al found that studies typically report around three PROMIS domains. ${ }^{38}$ Our study is the first that we are aware of to report and directly compare differences in these eight health domains across orthopaedic clinical subspecialties, providing baseline for PROMIS scores in orthopaedic. The direct comparisons reported in this paper would not be possible if using region-specific measures common to orthopaedic practice and research. By implementing PROMIS measures as a standard set of outcome measures, we can draw inferences about differences in patient-reported health status across orthopaedic populations that are typically not compared. Second, our study reported a clinical interpretation of PROMIS scores addressing the reported barrier to PROMIS use uptake. ${ }^{839}$ Providing a clinical interpretation is vital because often there is a disconnection between mean PROMIS scores (ie, physical functioning score 38.2) and how to interpret this information (moderate limitations in physical functioning). ${ }^{39}$

Our study is not without limitations. First, this was a cross-sectional cohort analysis, so we did not report PROMIS measures beyond baseline. Therefore, we cannot identify predictors of clinical outcomes or compare the change in PROMIS scores across subspecialities over time or downstream utilisation of orthopaedic procedures or rehabilitation services associated with baseline scores. Second, our findings may have limited generalisability. For example, the setting was a private hospital. It may not 
capture the broad diversity of non-white individuals in the area (79.3\% of individuals reported being Caucasian). Our instruments were only available in English, limiting data collection on non-English speaking patients. Moreover, in some clinical subspecialties reported (ie, trauma and orthopaedic oncology), small sample sizes potentially limit generalisability of these findings. Lastly, we did not compare PROMIS upper extremity physical function or legacy measures to our PROMIS measures as part of this study. Therefore, we cannot make direct comparisons of the performance of the eight reported PROMIS domains in this study to legacy or PROMIS upper extremity. However, the relationship between legacy measures in orthopaedics and PROMIS measures is well documented in the literature. ${ }^{40}$

Our study's findings are consistent with other literature regarding orthopaedic populations' physical and mental health status. ${ }^{41}$ In comparison to a study by Perruccio et al using the SF-36 as the outcome measure to physical and mental health in patients seeking care for musculoskeletal disorders, we found similar results where patients with spine disorders reported the most impairments in the cohort. ${ }^{41}$ Additionally, consistent with our results, they found hand upper extremity/hand patients were the healthiest and total joint arthroplasty patients demonstrated low levels of physical functioning. Our study's unexpected finding low levels of sleep disturbance, fatigue and depression reported across clinical subspecialties. Sleep disruption and pain frequently co-occur, both are uniquely linked with depressed mood ${ }^{42-45}$ and various forms of functional disability. ${ }^{46-48}$ Depression appears to play a substantial role in the sleep-pain linkage, particularly where the pain is severe. ${ }^{49}$ However, in our study, we did not find this relationship between pain, physical function, sleep disturbance and depression in the cohort, despite the research supporting these relationships. ${ }^{49}$

\section{CONCLUSIONS}

Reporting patients' health status consulting an orthopaedic provider using a standard set of outcome measures across various clinical subspecialties has numerous clinical care and research implications. Understanding the health status and clinical examination measures may improve patient and provider communication during the clinical encounter ${ }^{50}$ and be used as part of the prognostic evaluation. ${ }^{51}$ Moreover, this study can provide a context for informing bundled care or value-based care models. Classifying heterogeneous orthopaedic patients' baseline status on a standard metric could better inform the effectiveness and cost of treatment pathways. ${ }^{52}$ Lastly, reporting PROMIS scores has allowed the direct comparison of eight meaningful constructs across orthopaedic subspecialties. This comparison would not be possible with legacy measures, which is a noted strength of PROMIS measures. These comparisons allow unique insights to be made for orthopaedic departments and align clinical and research data collection with value-based care initiatives outside of orthopaedic departments. Clinicians and administrators can use this information to improve the delivery and the efficiency of care, improve and inform referral practices and inform subspecialty-specific education to improve patient outcomes from orthopaedic care.

\section{Author affiliations}

${ }^{1}$ Department of Orthopaedic Surgery, Duke University, Durham, NC, USA

${ }^{2}$ Department of Population Health Sciences, Duke University, Durham, NC, USA

${ }^{3}$ Duke Clinical Research Institute, Durham, North Carolina, USA

${ }^{4}$ Duke University, Durham, North Carolina, USA

\section{Twitter Maggie E Horn @onthehornside}

Acknowledgements The authors would like to acknowledge the Comprehensive Outcomes in Orthopaedics and Rehabilitation Data System group for supporting the infrastructure for data collection. The authors would also like to acknowledge Jennifer Gagnon for her contribution to data acquisition.

Collaborators Comprehensive Outcomes in Orthopaedics and Rehabilitation Data System group: Jennifer Gagnon.

Contributors MEH contributed to the work's conception, design of the study, data acquisition and writing of the manuscript. SZG contributed to the conception of the work, design of the manuscript's study and writing. EKR contributed to the conception of the work, design of the study, data acquisition and writing the manuscript. XY and SL contributed to the study's design and statistical analysis. MB contributed to the design of the manuscript's study and writing. BRR contributed to the design of the manuscript's study and writing. All authors approved the final manuscript.

Funding The authors have not declared a specific grant for this research from any funding agency in the public, commercial or not-for-profit sectors.

\section{Competing interests None declared.}

Patient and public involvement Patients and/or the public were not involved in the design, or conduct, or reporting, or dissemination plans of this research.

Patient consent for publication Not required.

Ethics approval This study was determined exempt by the Duke University Institutional Review Board (Pro00091740).

Provenance and peer review Not commissioned; externally peer reviewed.

Data availability statement Data are not available in accordance with protections provided by the HIPAA privacy information policy.

Open access This is an open access article distributed in accordance with the Creative Commons Attribution Non Commercial (CC BY-NC 4.0) license, which permits others to distribute, remix, adapt, build upon this work non-commercially, and license their derivative works on different terms, provided the original work is properly cited, appropriate credit is given, any changes made indicated, and the use is non-commercial. See: http://creativecommons.org/licenses/by-nc/4.0/.

\section{ORCID iD}

Maggie E Horn http://orcid.org/0000-0002-3963-7389

\section{REFERENCES}

1 Jones RS, Stukenborg GJ. Patient-reported outcomes measurement information system (PROMIS) use in surgical care: a scoping study. $J$ Am Coll Surg 2017;224:245-54.

2 Nelson EC, Eftimovska E, Lind C, et al. Patient reported outcome measures in practice. BMJ 2015;350:g7818.

3 Hung M, Stuart AR, Higgins TF, et al. Computerized adaptive testing using the PROMIS physical function item bank reduces test burden with less ceiling effects compared with the short musculoskeletal function assessment in orthopaedic trauma patients. J Orthop Trauma 2014;28:439-43.

4 Sikorskii A, Victorson D, O'Connor P, et al. PROMIS and legacy measures compared in a supportive care intervention for breast cancer patients and caregivers: experience from a randomized trial. Psychooncology 2018;27:2265-73.

5 PROMIS. Available: https://www.healthmeasures.net/score-andinterpret/interpret-scores/promis [Accessed 09 Jul 2020]. 
6 Kortlever JTP, Leyton-Mange A, Keulen MHF, et al. PROMIS physical function correlates with KOOS, jr in patients with knee pain. J Knee Surg 2020;33:903-11.

7 Moore MLG, Kortlever JTP, Keulen MHF, et al. PROMIS PF correlates with HOOS, jr in patients with hip pain. J Orthop 2020;21:58-61.

8 Brodke DJ, Saltzman CL, Brodke DS. PROMIS for orthopaedic outcomes measurement. J Am Acad Orthop Surg 2016;24:744-9.

9 Jildeh TR, Lizzio VA, Meta F, et al. The correlation between PROMIS pain interference and vas pain in ambulatory orthopedic patients. Orthopedics 2018:41:e813-9.

10 Cella D, Riley W, Stone A, et al. The patient-reported outcomes measurement information system (PROMIS) developed and tested its first wave of adult self-reported health outcome item banks: 2005 2008. J Clin Epidemiol 2010;63:1179-94.

11 Wilson I, Bohm E, Lübbeke A, et al. Orthopaedic registries with patient-reported outcome measures. EFORT Open Rev 2019;4:357-67.

12 Flynn KE, Dew MA, Lin L, et al. Reliability and construct validity of PROMIS $\otimes$ measures for patients with heart failure who undergo heart transplant. Qual Life Res 2015;24:2591-9.

13 Choi SW, Reise SP, Pilkonis PA, et al. Efficiency of static and computer adaptive short forms compared to full-length measures of depressive symptoms. Qual Life Res 2010;19:125-36.

14 Segawa E, Schalet B, Cella D. A comparison of computer adaptive tests (cats) and short forms in terms of accuracy and number of items administrated using PROMIS profile. Qual Life Res 2020;29:213-21.

15 Bruce B, Fries J, Lingala B, et al. Development and assessment of floor and ceiling items for the PROMIS physical function item bank. Arthritis Res Ther 2013;15:R144.

16 Amtmann D, Cook KF, Jensen MP, et al. Development of a PROMIS item bank to measure pain interference. Pain 2010;150:173-82.

17 Haefeli M, Elfering A. Pain assessment. Eur Spine J 2006;15 Suppl 1:S17-24.

18 Pilkonis PA, Yu L, Dodds NE. Validation of the depression item bank from the patient-reported outcomes measurement information system (PROMIS $®$ ) in a three-month observational. Journal of psychiatric 2014

19 Pilkonis PA, Choi SW, Reise SP, et al. Item banks for measuring emotional distress from the patient-reported outcomes measurement information system (PROMIS $\AA$ ): depression, anxiety, and anger. Assessment 2011;18:263-83.

20 Yu L, Buysse DJ, Germain A. Validation of the depression item bank from the patient-reported outcomes measurement information system (PROMIS) in a three-month observational study. Behav Sleep Med 2011;10:6-24.

21 Lai J-S, Cella D, Choi S, et al. How item banks and their application can influence measurement practice in rehabilitation medicine: a PROMIS fatigue item bank example. Arch Phys Med Rehabil 2011:92:S20-7.

22 Hahn EA, DeWalt DA, Bode RK, et al. New english and spanish socia health measures will facilitate evaluating health determinants. Health Psychol 2014:33:490-9.

23 Hahn EA, Devellis RF, Bode RK, et al. Measuring social health in the patient-reported outcomes measurement information system (PROMIS): item bank development and testing. Qual Life Res 2010;19:1035-44.

24 Rothrock NE, Hays RD, Spritzer K, et al. Relative to the general US population, chronic diseases are associated with poorer healthrelated quality of life as measured by the patient-reported outcomes measurement information system (PROMIS). J Clin Epidemiol 2010;63:1195-204

25 Ripley BD. The R project in statistical computing. MSOR Connections The newsletter of the LTSN Maths : $2001 \mathrm{http}: / /$ citeseerx.ist.psu.edu/viewdoc/download?doi=10.1.1.430.3979\&rep= rep1\&type =pdf

26 Score Cut Points. Available: https://www.healthmeasures.net/ score-and-interpret/interpret-scores/promis/promis-score-cut-points [Accessed 27 Oct 2020].

27 Mukaka MM. Statistics corner: a guide to appropriate use of correlation coefficient in medical research. Malawi Med $J$ 2012;24:69-71.

28 Von Korff M, Scher Al, Helmick C, et al. United States national pain strategy for population research: concepts, definitions, and pilot data. J Pain 2016;17:1068-80.

29 St Sauver JL, Warner DO, Yawn BP, et al. Why patients visit their doctors: assessing the most prevalent conditions in a defined American population. Mayo Clin Proc 2013;88:56-67.
30 Makhni EC, Meadows M, Hamamoto JT, et al. Patient reported outcomes measurement information system (PROMIS) in the upper extremity: the future of outcomes reporting? J Shoulder Elbow Surg 2017;26:352-7.

31 Beleckas CM, Prather H, Guattery J, et al. Anxiety in the orthopedic patient: using PROMIS to assess mental health. Qual Life Res 2018;27:2275-82.

32 McCrabb S, Baker AL, Attia J, et al. Comorbid tobacco and other substance use and symptoms of anxiety and depression among hospitalised orthopaedic trauma patients. BMC Psychiatry 2019;19:28

33 Perruccio AV, Davis AM, Hogg-Johnson S, et al. Importance of selfrated health and mental well-being in predicting health outcomes following total joint replacement surgery for osteoarthritis. Arthritis Care Res 2011;63:973-81 https://onlinelibrary.wiley.com/doi/abs/

34 Hopman WM, Towheed T, Anastassiades T. Canadian normative data for the SF-36 health survey. CMAJ 2000;163:265-71 https://www. cmaj.ca/content/163/3/265.short

35 Raad M, Amin RM, El Abiad JM, et al. Preoperative patient functiona status is an independent predictor of outcomes after primary total hip arthroplasty. Orthopedics 2019;42:e326-30.

36 Lyman S, Lee Y-Y, Franklin PD, et al. Validation of the KOOS, jr: a short-form knee arthroplasty outcomes survey. Clin Orthop Relat Res 2016:474:1461-71.

37 Gulledge CM, Lizzio VA, Smith DG, et al. What are the floor and ceiling effects of patient-reported outcomes measurement information system computer adaptive test domains in orthopaedic patients? A systematic review. Arthroscopy 2020;36:901-12.

38 Horn ME, Reinke EK, Couce LJ, et al. Reporting and utilization of patient-reported outcomes measurement information System ${ }^{\circledR}$ (PROMIS $($ ) measures in orthopedic research and practice: a systematic review. J Orthop Surg Res 2020;15:553.

39 O'Hara NN, Richards JT, Overmann A, et al. Is PROMIS the new standard for patient-reported outcomes measures in orthopaedic trauma research? Injury 2020;51 Suppl 2:S43-S50.

40 Cheung EC, Moore LK, Flores SE, et al. Correlation of PROMIS with orthopaedic patient-reported outcome measures. JBJS Rev 2019;7:e9.

41 Perruccio AV, Gandhi R, Rampersaud YR, et al. Heterogeneity in health status and the influence of patient characteristics across patients seeking musculoskeletal orthopaedic care - a crosssectional study. BMC Musculoskelet Disord 2013;14:83.

42 Chen Q, Hayman LL, Shmerling RH, et al. Characteristics of chronic pain associated with sleep difficulty in older adults: the maintenance of balance, independent living, intellect, and Zest in the elderly (mobilize) Boston study. J Am Geriatr Soc 2011;59:1385-92.

43 McCurry SM, Von Korff M, Vitiello MV, et al. Frequency of comorbid insomnia, pain, and depression in older adults with osteoarthritis: predictors of enrollment in a randomized treatment trial. $J$ Psychosom Res 2011;71:296-9.

44 Smith MT, Quartana PJ, Okonkwo RM, et al. Mechanisms by which sleep disturbance contributes to osteoarthritis pain: a conceptual model. Curr Pain Headache Rep 2009;13:447-54.

45 Cole JC, Dubois D, Kosinski M. Use of patient-reported sleep measures in clinical trials of pain treatment: a literature review and synthesis of current sleep measures and a conceptual model of sleep disturbance in pain. Clin Ther 2007;29 Suppl:2580-8.

46 Goldman SE, Stone KL, Ancoli-Israel S, et al. Poor sleep is associated with poorer physical performance and greater functional limitations in older women. Sleep 2007;30:1317-24.

47 Guccione AA, Felson DT, Anderson JJ, et al. The effects of specific medical conditions on the functional limitations of elders in the Framingham study. Am J Public Health 1994;84:351-8.

48 McCracken LM, Iverson GL. Disrupted sleep patterns and daily functioning in patients with chronic pain. Pain Res Manag 2002:7:75-9.

49 Finan PH, Goodin BR, Smith MT. The association of sleep and pain: an update and a path forward. J Pain 2013;14:1539-52.

50 Olde Rikkert MGM, van der Wees PJ, Schoon Y, et al. Using patient reported outcomes measures to promote integrated care. Int $J$ Integr Care 2018;18:8.

51 Van Der Wees PJ, NIJHUIS-VAN DER SANDEN MWG, AYANIAN $\mathrm{JZ}$, et al. Integrating the use of patient-reported outcomes for both clinical practice and performance measurement: views of experts from 3 countries. Milbank Q 2014;92:754-75.

52 Porter ME. What is value in health care? N Engl $\mathrm{J} \mathrm{Med}$ 2010;363:2477-81. 